\title{
The Biogenic Stimulator for Nonspecific Immunity Provocation in the Experiment "in vivo" in the Stress Condition
}

\author{
Grebenshchikov A.V.* Vasilenko L.I. Ozherelyeva O.N. \\ Mal'tseva O.U. Rogov K.V. Lopatina A.A. \\ Voronezh State University of Engineering Technologies, 19, Revolutsii ave., Voronezh, 394036, Russia. \\ *Corresponding author. Email: serafim10@yandex.ru
}

\begin{abstract}
A biogenic stimulant (Patent RU 2648466) was used as a study subject. Comparison objects are Ligfolum and Spleninum. Nonspecific resistance of experimental animals was estimated with the use of some leucocytal indices: the Krebs index (KI), leucocytal index of intoxication (LII), white blood cells dislocation index (WBCDI) - according to Jabluchanskij A.A., leukocytal index (LI). It was established that induced stress influenced directly by the number of leucocytes. Induced stress also had an impact on the blood leucogram of the animals undergoing the stress and taking part in the experiment. Leucocytal indices were figured out for animals' complex immunological status in dedication of the stress condition and immunomodulators application. Krebs index, which is the ratio between neutrophilic leucocyte and lymphocyte cells, characterizes indirectly an activity level of phagocytal reactions and non-specific immune system factors, as well as their involvement in general organism reactivity support. A ratio of the neutrophilic leucocyte level and the amount of lymphocyte cells, monocytes and eosinophils characterize Leucocytal index of intoxication (LII), characterize the activity level of phagocytosis and neutrophilic leucocyte proliferation. During the experiment, white blood cells dislocation index (WBCDI) characterizes the ratio between granular leucocyte and agranular leukocyte. The ratio between lymphocytes and neutrophils is described by leukocytal index (LI), which characterizes the ratio between humoral and cellulated elements of the experimental animals' immune system. Relations between the affecter and effector elements of the immunological process are characterized by lymphocytes and monocytes ratio index (LMRI), which describes the balance between lymphocytes and monocytes.
\end{abstract}

\section{Keywords: biogenic stimulant, stress condition, nonspecific immunity provocation}

\section{INTRODUCTION}

During several past decades immune therapy has become the clinical reality; more and more patients are getting immunologic treatment. Medical attention is focused on the developing of the ways helping to provoke and strengthen the current immune response against different disease-producing factors [1-5].

Fast development of immunology during the recent years has significantly increased our knowledge about protection mechanisms of mammals' organisms [6, 7]. Molecular mechanisms of cellular interaction during the immune response have been discovered, as well as intracellular responses included in signal transmission. Also the considerable number of immune and inflammatory reactions transmitters has been revealed $[1,3,8]$.

The first result of the knowledge development in this field gave an opportunity to researchers and veterinary specialists to explore immunological basis of illness processes with the help of the pharmacotherapeutic tools battery; at the same time this result facilitated early recovery of the animal's productive health. It has made diagnosis of diseases much more accurate and allowed doctors to adapt the therapy to the particular patient's needs $[8,11]$. However, better understanding of illness processes provides only gradual therapy improvement. It is caused by the fact that new discovered molecular targets must be checked as potential motifs for immunomodulating effect from pharmaceutical drugs and then new compounds must become the object of extended development [10]. At the moment in the science it is absolutely clear to a certain degree how some well-known treatment methods, such as immunomodulating, antiallergic and antiasthmatic medicines (including corticosteroids), work. Some new progressive methods of treatment, based on molecular mechanisms, are also currently being used in clinical practice and they are contributing for cancer treatment and against infectious and autoimmune diseases $[12,13]$. 
Together with these achievements in understanding of immune molecular mechanisms, immunodiagnosis and immunotherapy, it has become possible to check more accurately how different medicine classes interrelate to the immune system. It is especially important for regulating organs to distinguish toxical side effects of immunomdulating medicines from their useful therapy effects [14].

Current non-clinical researches are checking safety and effectiveness of biological, molecular, cellular and histological pharmaceutical chemicals either as monotherapy or together with conventional management of treatment.

Immunomdulating medicines have been used in medicine for treatment and as preventive measure since the beginning of the XX century, William B. Coley gave treatment to oncologic patients with mixed bacteria toxins [5-7]. These studies have stimulated the clinical use of Prodigiozan, Timolin, Ligfolum, Biostim, BronchoVaxom (recurrent infections, Europe) and many others medicines. Usage of these medicines is limited by some factors because they can induce different immunopharmocologic effects [16].

Similarly, biogenous pharmaceutical stimulants can be the source of the active ingredients for immune therapy, they require clearance, characterization and production of the active components. Made of natural products refined objects, which are used in "routine" common clinical practice, are included into Spleninum, Ligfolum, produced with natural components [13-15]. Nowadays a lot of attention is paid to the use of biogenous stimulants in immune therapy, though the useful effect of these medicines can be limited by their immune toxicity and pharmacological deficiencies. However, modificators of biological response are potentially efficient because of their ability to induce multiple cytokines for immune augmentation and hematosis recovery [17].

Among various productive animals immunopathologies are immunodeficiency disorders such as primary, age-related and secondary (adult-onset), which are widely spread and are of great importance [18].

As veterinary practice shows, young productive animals have immunodeficiency disorders as a result of insufficient and untimely colostric and transovarian protection factors intake and the immune system immaturity [13].

Pathogenous influence of various environmental factors causes young productive animals' symptom complex, leading to the development of ecologically associated secondary immune deficiency conditions [14]. Immunopathology data is connected with lymphoid organ function. As a result reasonable diagnostic assessment of immunodeficiency disorders should be made with the help of immunomorphologic methods as they allow to determine the immunocompetent system condition and to infer about the immune deficiency degree according to morphofunctional changes $[13,14]$

It must be mentioned that the problem of after immune disorders rehabilitation with immunomodulator treatment is currently important as most of factor and contagious animal diseases are accompanied by secondary immune deficiency disorders [15-17]. One of the examples of such immunomodulators is biogenous stimulant which action is based on the immunocompetent organs activation $[15,16]$. As a result it has become absolutely essential to study clinicopathologic feature of immune pathology in the experiment "in vivo" on white inbred mice BALB/C line and its correction with biogenous stimulant.

\section{THE OBJECT OF THE STUDY}

As an object of research Biogenous stimulant (Patent RU 2648466) was used. Comparison objects were Ligfolum and Spleninum. Ligfolum as an active substance contains humic compounds, made with natural (wood spirit) lignin hydrolysis. It also includes pyrophosphates decahydrate, sodium chloride and demineralised water. It does not obtain pyretogenetic, allergic, teratogenic and embryotoxicity effects. Ligfolum is effective for resistance increase, reduces negative stress consequences, immunodefence increases during various diseases, including neoplastic diseases. Spleninum is a biologically active medicine made of cattle spleen with extraction.

Biogenous stimulant (Patent RU 2648466) is a biologically active medicine made of cattle milts. It is limpid, light yellow liquid, salty with specific smell; $\mathrm{pH}$ 4,0-5,0. According to experimental data, Biogenous stimulant (Patent RU 2648466) is able to stimulate reparative processes, improves nitrogen metabolism recovery, obtains adaptogene stress-correction kinesis. Valeopositive effects of Biogenous stimulant (Patent RU 2648466) are based on immune antioxidant mechanisms, including anti eradicative activity, phagocytic cell mobilization, antioxidant protection and immunocompetent organs activation. Biogenous stimulant (Patent RU 2648466) is low-toxic medicine.

\section{MATERIALS AND METHODS}

Experiment worksheet, animals maintenance and taking them out of the experiment were held according to bioethics fundamentals of European Community Declaration (86/609/ECD) and All-Union standard P 53434-2009 "Laboratory practice proper principles". The research had been held on white inbred mice BALB/C line, 21 days old. Mice were held in common vivarium conditions. Before the beginning and after the ending of the experiment in order to indicate the stress resistance of experimental animals the method "open field" 5-minutes test was carried out and indicant "resistance coefficient" (CR) was measured [18]. Depending on the behavior during "open field" test, all animals were divided into two groups where researching indices statistically differed. The first group animals were denoted as "active" stressresistant (SR) (CR $=2.005 .00)$, the second group animals 
- "passive" stress-nonresistant $(\mathrm{SN})(\mathrm{CR}=0.30-0.70)$. "Passive" group animals were treated with inveterate interrupted immobilization, which was simulated with the help of rigid fixation of the animals' 4 extremities stomach upwards to research-oriented machine during 60 minutes daily for 10 days. The animals were randomly divided into 5 groups, with 30 animals in each one: group 1 - intact animals without caused stress and without immunomodulator injection; group 2 - control animals with provoked stress; group 3 - animals with provoked stress and Biogenous stimulant injections; group 4 animals with provoked stress and Spleninum injections; group 5 - animals with provoked stress and Ligfolum injections. Experimental animals and animals from control groups were being taken out of the experiment with sodium pentobarbital injection in dose $60 \mathrm{mg} / \mathrm{kg}$ abdominally, as well as blood sampling from cardial cavities had been led [18]. Experimental animals' nonspecific resistance was estimated with the use of some leucocytal indices: the Krebs index (KI), leucocytal index of intoxication (LII), white blood cells dislocation index (WBCDI) - according to Jabluchanskij A. A., leukocytal index (LI) $[18,19]$. Statistical analysis was held every ten days by the program QtiPlot. Statistical data accuracy is set for independent samples based on U-criteria MannWhitney. Spirman rank test had been used for correlation dependence identification. Samples differs were considered evidential with significance point $\mathrm{p} \leq 0.05$.

\section{DISCUSSION OF THE RESULTS}

Leucocytes play one of the main roles in homeostasis conservation of the animal organism, as they take part in deactivation of contagiums either exogeneous or autogenous genesis. During the experiment it was established that induced stress was having direct impact on the overall number of leucocytes (table 1). For example, leucocytes level of the animals in group 2 (control animals with provoked stress and without immunomodulator injection) had decreased by $29 \%$. Leucocytes level of the animals in group 3 (animals with provoked stress and Biogenous stimulant injections) had decreased by $6.5 \%$. Leucocytes level of the animals in group 4 (animals with provoked stress and Spleninum injections) had decreased for $3.2 \%$, which was the minimum result. Leucocytes level of the animals in group 5 (animals with provoked stress and Ligfolum injections) had decreased by $12.9 \%$. Leucocytes level of the intact animals (group 1) had not changed.

Table 1 Blood leukogram of laboratory animals during stress provocation and immunomodulator treatment

\begin{tabular}{|c|c|c|c|c|c|c|}
\hline Group & $\begin{array}{c}\text { Leucocytes, } \\
109 / 1, \mathrm{M} \pm \mathrm{m}\end{array}$ & $\begin{array}{c}\text { Lymphocytes, } \\
\%, \mathrm{M} \pm \mathrm{m}\end{array}$ & $\begin{array}{c}\text { Monocytes, } \\
\%, \mathrm{M} \pm \mathrm{m}\end{array}$ & $\begin{array}{c}\text { Neutrophils, } \\
\%, \mathrm{M} \pm \mathrm{m}\end{array}$ & $\begin{array}{c}\text { Eosinophils, \%, } \\
\mathrm{M} \pm \mathrm{m}\end{array}$ & $\begin{array}{c}\text { Basophiles, } \\
\%, \mathrm{M} \pm \mathrm{m}\end{array}$ \\
\hline \multicolumn{7}{|c|}{ Before stress provocation and immunomodulator treatment } \\
\hline 2 & $3,0 \pm 0,3$ & $71 \pm 5$ & $1,6 \pm 0,11$ & $29 \pm 2,3$ & $1,2 \pm 0,11$ & $0,6 \pm 0,09$ \\
\hline 3 & $3,1 \pm 0,3$ & $70 \pm 6$ & $1,5 \pm 0,10$ & $27 \pm 2,5$ & $1,2 \pm 0,11$ & $0,4 \pm 0,09$ \\
\hline 4 & $3,2 \pm 0,3$ & $68 \pm 5$ & $1,9 \pm 0,11$ & $28 \pm 3,8$ & $1,1 \pm 0,10$ & $0,6 \pm 0,07$ \\
\hline 5 & $3,1 \pm 0,3$ & $69 \pm 5$ & $1,7 \pm 0,17$ & $29 \pm 3,7$ & $1,3 \pm 0,11$ & $0,4 \pm 0,08$ \\
\hline & & $69 \pm 5$ & $1,7 \pm 0,12$ & $29 \pm 4,1$ & $1,2 \pm 0,10$ & $0,6 \pm 0,06$ \\
\hline 1 & $3,0 \pm 0,3$ & $71 \pm 5$ & $1,5 \pm 0,10$ & $28 \pm 2,4$ & $1,2 \pm 0,10$ & $0,6 \pm 0,07$ \\
\hline 2 & $2,2 \pm 0,3$ & $43 \pm 9$ & $0,4 \pm 0,09$ & $21 \pm 2,5$ & $1,0 \pm 0,09$ & $0,3 \pm 0,07$ \\
\hline 3 & $2,9 \pm 0,3$ & $58 \pm 6$ & $0,7 \pm 0,10$ & $26 \pm 2,2$ & $1,1 \pm 0,09$ & $0,4 \pm 0,06$ \\
\hline 4 & $3,0 \pm 0,3$ & $68 \pm 6$ & $0,8 \pm 0,05$ & $28 \pm 2,2$ & $1,1 \pm 0,10$ & $0,5 \pm 0,08$ \\
\hline 5 & $2,7 \pm 0,3$ & $55 \pm 7$ & $0,7 \pm 0,11$ & $25 \pm 2,4$ & $1,0 \pm 0,09$ & $0,4 \pm 0,07$ \\
\hline
\end{tabular}


Induced stress also influenced the blood leukogram of the experimental animals suffered from stress conditions (table 1).

Obtained data analysis has showed that significant decrease was presented by lymphocytes, monocytes and basophiles. Blood leukogram of controlled animals was featured with maximum low numbers, for instance, in comparison with intact animals lymphocytes the level was lower by $39.4 \%$, monocytes - by $18.8 \%$, neutrophils - by $27.6 \%$, eosinophils and basophiles - by 16.7 and $50 \%$.

Blood leukogram of the animals from group 4 (animals with provoked stress and Spleninum injections) had the minimum (the lowest) changes. For instance, in comparison with intact animals lymphocytes level was the lowest by $4.2 \%$, monocytes - by $6.3 \%$, neutrophils - by $3.4 \%$, eosinophils and basophiles - by $8.3 \%$ and $16.7 \%$ in accordance.

Comparative data of blood leukogram of the animals from group 3 (animals with provoked stress and Biogenous stimulant injections) had showed the following results: in comparison with intact animals lymphocytes level was lower by $18.3 \%$, monocytes - by $6.3 \%$, neutrophils - by $6.9 \%$, eosinophils and basophiles - by 8.3 and $33.3 \%$.

For complex immunological status identification of the animals in stress condition and with immunomodulator treatment leukocytic indices had been calculated (table 2):

Krebs index, which is the ratio between neutrophilic leucocyte and lymphocyte cells, characterizes indirectly the activity level of phagocytal reactions and non-specific immune system factors, as well as their involvement in general organism reactivity support. During the experiment Krebs the index amount was increasing by $19.1 \%$ in group 2 (controlled animals with provoked stress and without immunomodulator injection). In group 3 (animals with provoked stress and Biogenous stimulant injections) the increase was $13.5 \%$. In group 4 (animals with provoked stress and Spleninum injections) as well as in group 1 (intact animals) no evidential changes of this feature were noticed. In group 5 (animals with provoked stress and Ligfolum injections) the increase of the index was $15.3 \%$. Probably, these results were connected with functional oppression of immunocompetent organs proliferative capability.

Table 2 Blood leukogram of laboratory animals during stress provocation and immunomodulator treatment

\begin{tabular}{|c|c|c|c|c|c|}
\hline Group & Krebs index & LII & WBCDI & LI & LMRI \\
\hline \multicolumn{6}{|c|}{ Before stress provocation and immunomodulator treatment } \\
\hline 1 & $0,41 \pm 0,111$ & $0,39 \pm, 111$ & $0,42 \pm, 111$ & $2,45 \pm 0,459$ & $44,4 \pm 2,23$ \\
\hline 2 & $0,39 \pm 0,108$ & $0,37 \pm 0,134$ & $0,40 \pm 0,109$ & $2,59 \pm 0,538$ & $46,7 \pm 2,46$ \\
\hline 3 & $0,41 \pm 0,112$ & $0,39 \pm 0,127$ & $0,42 \pm 0,127$ & $2,43 \pm 0,399$ & $35,8 \pm 2,33$ \\
\hline 4 & $0,42 \pm 0,112$ & $0,40 \pm, 111$ & $0,44 \pm 0,112$ & $2,38 \pm 0,458$ & $40,6 \pm 2,87$ \\
\hline 5 & $0,42 \pm 0,125$ & $0,40 \pm 0,112$ & $0,43 \pm 0,127$ & $2,38 \pm 0,535$ & $40,6 \pm 4,33$ \\
\hline \multicolumn{6}{|c|}{ After stress provocation and immunomodulator treatment } \\
\hline 1 & $0,41 \pm 0,109$ & $0,38 \pm 0,112$ & $0,41 \pm 0,140$ & $2,54 \pm 0,546$ & $47,3 \pm 2,91$ \\
\hline 2 & $0,49 \pm 0,127$ & $0,47 \pm 0,089$ & $0,51 \pm, 111$ & $2,05 \pm 0,447$ & $107,5 \pm 8,47$ \\
\hline 3 & $0,47 \pm 0,212$ & $0,43 \pm, 111$ & $0,47 \pm 0,075$ & $2,23 \pm 0,478$ & $82,9 \pm 4,23$ \\
\hline 4 & $0,41 \pm 0,134$ & $0,42 \pm, 111$ & $0,46 \pm 0,127$ & $2,29 \pm 0,551$ & $80,0 \pm 2,19$ \\
\hline 5 & $0,47 \pm 0,141$ & $0,44 \pm 0,109$ & $0,47 \pm 0,112$ & $2,20 \pm 0,399$ & $78,6 \pm 4,38$ \\
\hline
\end{tabular}

The ratio of the neutrophilic leucocyte level and the amount of lymphocyte cells, monocytes and eosinophils characterizes Leucocytal index of intoxication (LII), characterizes the activity level of phagocytosis and neutrophilic leucocyte proliferation. During the experiment in group 2 (controlled animals with provoked 
stress and without immunomodulator injection) the level of LII had been increasing by $18.9 \%$. In group 3 (animals with provoked stress and Biogenous stimulant injections) the level of LII had been increasing by $14.2 \%$. In group 4 (animals with provoked stress and Spleninum injections) as well as in group 1 (intact animals) no evidential changes of this index were noticed. In group 5 (animals with provoked stress and Ligfolum injections) the level of LII had been increasing by $16.1 \%$. Discovered changes of leucocytal index of intoxication reflected the presence of endogenic toxicosis.

During the experiment level of WBCDI, showing ratio of granulocytes and agranulocytes, in group 2 (controlled animals with provoked stress and without immunomodulator injection) increased by $19.9 \%$. In group 3 (animals with provoked stress and Biogenous stimulant injections) the level of WBCDI had been increasing by $14 \%$. In group 4 (animals with provoked stress and Spleninum injections) as well as in group 1 (intact animals) no evidential changes of this index were noticed. In group 5 (animals with provoked stress and Ligfolum injections) the level of WBCDI had been increasing by $15.7 \%$. Discovered changes of WBCDI showed immune responsiveness disorder of the experimental animals' organisms.

Ratio between lymphocytes and neutrophils is described by leukocytal index (LI), which characterizes ratio between humoral and cellulated elements of the experimental animals' immune system. In group 2 (controlled animals with provoked stress and without immunomodulator injection) the level of LI decreased for $20.9 \%$. In group 3 (animals with provoked stress and Biogenous stimulant injections) the level of LI decreased by $17.1 \%$. In group 4 (animals with provoked stress and Spleninum injections) as well as in group 1 (intact animals) no evidential changes of this index were noticed. In group 5 (animals with provoked stress and Ligfolum injections) the level of LI increased by $18.3 \%$. Discovered changes of LI level also demonstrated the presence of functional oppression of immunocompetent organs proliferative capability.

Relations between affecter and effector elements of immunological process are characterized by lymphocytes and monocytes ratio index (LMRI), which describes the balance between lymphocytes and monocytes. LMRI level during the experiment in group 2 (controlled animals with provoked stress and without immunomodulator injection) decreased for $29.2 \%$. In group 3 (animals with provoked stress and Biogenous stimulant injections) the level of LMRI decreased for $17.2 \%$. In group 4 (animals with provoked stress and Spleninum injections) as well as in group 1 (intact animals) no evidential changes of this index were noticed. In group 5 (animals with provoked stress and Ligfolum injections) the level of LMRI increased for $15.9 \%$. Discovered changes of LMRI demonstrated the presence of monocytopenia, which is beneficial in functional activity of the lymphocytic system support in the setting of general autoimmune processes in the experimental animals' organisms.

\section{CONCLUSION}

On the base of analyzed results, made during the experiment, it was established that induced stress had a direct impact on the general amount of leucocytes. Dramatic reduction of lymphocytes, monocytes and basophiles had been noticed. Minimal changes showed blood leukogram of the animals from group 4 (with provoked stress and Spleninum injections).

Comparative data of blood leukogram was got of group 3 (animals with provoked stress and Biogenous stimulant injections). In the setting of induced stress experimental groups animals showed such disorders as: functional oppression of immunocompetent organs proliferative capability, endogenic toxicosis, immune responsiveness disorder of the experimental animals' organisms. Discovered changes of LI level also demonstrated the presence of functional oppression of immunocompetent organs proliferative capability. Discovered changes of LMRI demonstrated the presence of monocytopenia, which is beneficial in functional activity of the lymphocytic system support in the setting of general autoimmune processes in the experimental animals ' organisms.

It must be mentioned that immunomodulator Spleninum had protective capability for immunocompetent organs of the animals in stress conditions as well as supported cellulated resistance at the proper level. At the same time biogenic stimulant (Patent RU 2648466) showed the comparative data with Spleninum medicine.

\section{REFERENCES}

[1] J.E. Talmadge, Immunostimulants in Cancer Therapy, Nijkamp and Parnham's Principles of Immunopharmacology, Springer, Cham, 2019.

[2] F. Puggioni, M. Alves-Correia, M. Mohamed et al., Immunostimulants in respiratory diseases: focus on Pidotimod, Multidiscip Respir. Med. 14 (2019) 31. Retrieved from: https://doi.org/10.1186/s40248-0190195-2

[3] J.G. Pol, P. Caudana, J. Paillet et al., Effects of interleukin-2 in immunostimulation and immunosuppression, J. Exp. Med. 217(1) (2020) e20191247. DOI: https://doi.org/10.1084/jem.20191247

[4] U. Studer, E. Marti, D. Stornetta et al., Therapy of equine sarcoid with a nonspecific immunostimulator - the epidemiology and spontaneous regression of sarcoids, Schweizer Archiv für Tierheilkunde 139 (1997) 385-391.

[5] J.T. Miura, J.S. Zager, Intralesional Therapies: Oncolytic Viral Therapies, Immunostimulants, Cancer Regional Therapy, Springer, Cham, 2020. Retrieved from: https://doi.org/10.1007/978-3-03028891-4_35 
[6] K.K. Vijayan, Compendium on Prophylaxis in Aquaculture, ICAR - Central Inst. of Brackishwater Aquacult., Indian Council of Agricult. Res., 2017.

[7] J.G. Pol, P. Caudana, J. Paillet, E. Piaggio, Guido Kroemer Effects of interleukin-2 in immunostimulation and immunosuppression, J. Exp. Med. 217(1) (2020) e20191247. Retrieved from: https://doi.org/10.1084/jem.20191247

[8] L. Eileen, Thacker Immunomodulators, immunostimulants, and immunotherapies in small animal veterinary medicine, Veter. Clin. of North Amer.: Small Animal Pract. 40(3) (2010) 473-483.

[9] L.M. Hang, M.T. Aguado, F.J. Dixon, Theofilopoulos A.N. Induction of severe autoimmune disease in normal mice by simultaneous action of multiple immunostimulators, J. Exp. Med. 161(2) (1985) 423-428. Retrieved from: https://doi.org/10.1084/jem.161.2.423

[10] S.S. Konda, O.Y. Parasuraman, S.R. Aiya, Dinakaran Michael Polysaccharide fraction from the Indian mistletoe, Dendrophthoe falcata (L.f.) Ettingsh enhances innate immunity and disease resistance in Oreochromis niloticus (Linn.), Fish \& Shellfish Immunol. 88 (2019) 407-414.

[11] D. Usmanov, N.Sh. Ramazanov, U. Yusupova, K.Dzh. Kucherbayev, Iridoids from phlomis severtzovii and its immunostimulating and antitoxic activity, News of the National Acad. of Sci. of the Republic of Kazakhstan. Ser. of chem. and technol. 6(438) (2019) 49-54.

[12] E.P. Simurzina, V.G. Semenov, The optimization of reproductive and productive qualities of cattle by native immunostimulants, Sci. notes of Kazan Bauman state acad. of veter. Med. 240(IV) (2019) 180-187.
[13] A. Nawaza, A. Bakhsh javaida, S.I. Seyed et al., The functionality of prebiotics as immunostimulant: Evidences from trials on terrestrial and aquatic animals, Fish \& Shellfish Immunol. 76 (2018) 272-278.

[14] A Turmagambetova, A Bogoyavlenskiy, V Berezin et al., Composite immunostimulants is a new way for immune system regulation, J. of Biotechnol. 305 (2019) S55.

[15] A.V. Grebenshchikov, L.I. Vasilenko, A.U. Korchagina, Research of the influence of biogenic stimulant in the experiment "in vivo" on white inbred mice BALB/C line, Innovations in agriculture, veterinary and food industry. Collection of scientific articles of the 84-th scientific-practical conference, 2019, pp. 529-531.

[16] A.V. Grebenshchikov, L.I. Vasilenko, A.E. Kucova, A.I. Golubev, RU, Patent No. 2648466, The way of getting biogenic stimulant for treatment and preventative medication of agricultural animals' diseases, 2016.

[17] A V Grebenshchikov, L I Vasilenko, A E Kucova et al., Optimization of biogenic stimulant active substances forming performance in animal's tissues before extraction test, Innovations in food industry: science, education and production, IV International correspondence science - technical conference materials, Voronezh State Univer. of Engineer. Technol., 2017, pp. 237-240.

[18] E.V. Koplik, Method of identification rat resistance to emotional stress criteria, News of medical technologies 9(1) (2002) 16-18.

[19] E.A. Tkachenko, M.A. Derho, Leucocytal indices during mice experimental cadmium intoxication, Izv.

OGAU 3 (2014) 81-83. 